\title{
A comprehensive review for optimal placement of phasor measurement unit for network observability
}

\author{
Maveeya Baba ${ }^{1}$, Nursyarizal B. M. Nor ${ }^{2}$, Taib B. Ibrahim ${ }^{3}$, M. Aman Sheikh ${ }^{4}$ \\ 1,2,3 Department of Electrical and Electronics Engineering, Universiti Tecknologi PETRONAS, Malaysia \\ ${ }^{4}$ Department of Computing and Information System, Sunway University, Malaysia
}

\begin{tabular}{l}
\hline Article Info \\
\hline Article history: \\
Received Nov 11, 2019 \\
Revised Jan 10, 2020 \\
Accepted Jan 28, 2020 \\
\hline
\end{tabular}

\section{Keywords:}

Optimal placement problem of PMUs (OPPP)

Phasor measurement units (PMUs)

State estimation (SE)

\begin{abstract}
Real time synchronized phasor measurement in power network is obtained by the improvement in monitoring, control and, protection of the power system. In recent time, the installation ratio of phasor measurement units (PMUs) is constantly increasing for the real time measurement throughout worldwide. The increment in the number of PMU installation is to only focus on the improvement of system state estimation (SE) performance. However, the expensive nature of the metering device requires huge amount of installation cost with the other communication facilities; therefore, an optimal placement of PMU is necessary. Different techniques have been designed and used to overcome this matter. The paper presents numerous optimization algorithms such as, Mathematical programming, Heuristic, and Meta-Heuristic techniques which are specially used for the optimization of PMU placement with complete network observability. Furthermore, each PMU technique is explained, and performances are compared for the most appropriate and optimal placement of PMU methods, which can be recommended for a future work to get complete network observability.
\end{abstract}

Copyright $(92020$ Institute of Advanced Engineering and Science. All rights reserved.

\section{Corresponding Author:}

Maveeya Baba,

Department of Electrical and Electronics Engineering,

Universiti Tecknologi PETRONAS,

Malaysia.

Email: Maveeya18000611@utp.edu.my

\section{INTRODUCTION}

The conventional measurements that control, protect and monitor the power network are based on the local estimation. However, if the traditional approaches are utilized to monitor, control and protect the whole network, then it is thoroughly difficult to retain the reliability, performance, and secure the system as a whole [1]. In current scenario the power system is generally linked with the conventional meters and are planning towards the installation of most precise device phasor measurement units (PMUs) to secure, control, and monitor the power network [2]. The conventional methods which measure the power flow, voltage phasors and current phasors of the buses are too repetitive and immense in nature [3]. In 2003, different number of regions in USA and Canada were suffered due to the outage of power supply, later they called it large blackout. In-fact, 55 millions of people were badly affected because of the shortage of electricity and it is considered as a ninth largest power outage in the world. Afterwards in February 2004, the US-Canada outage task force made a report about the incident which disturbed the power utilities and transmission lines [4]. Finally, they submitted a report to the government officials. Besides, they recommended the use of PMU to get better visibility of power grids and avoid such incidents in future. Before there were few PMUs installed in the power network, after that incident (Blackout) number of PMUs are increased from 166 in 2010 to 1126 in 2014 [4]. 
Power system state estimation (PSSE) is conventionally a nonlinear problem, yet PMUs take the measurement and formulate it as a linear problem, it is easy to solve linear properties which are much simpler than nonlinear features [5]. It is identified that the conventional PSSE technique is not reliable in convergence speed and is inefficient in measurement for a wide area network. PMUs are directly linked with global positioning system (GPS), which is useful in power system's stability and SE calculation. It contributes a new method and a solution to overcome the above-mentioned issues as PMUs can continuously monitor the maximum value of voltage and current waveforms. Also, it has a capability of measuring phase angles of the strategical located buses with high accuracy in power system [6]. Meanwhile, GPS provides small amount of synchronization error, the question arises on which place and in what way PMUs need to be placed in a power system with the low cost of communication facilities and maximum observability of power network. The concept is known as optimal pmu placement (OPP) [7].

A power system is supposed to be measurable when all the parameters of its buses are known. Obviously, once PMUs take the measurement of all the buses in power grid, and the measurement of all the PMUs are directly interconnected with the wide area measurement centres through GPS. Hence, the current phasor which flows through all the incident branches and voltage phasor at all the buses where PMUs are equipped would be identified. This new mechanism has potentially transformed the old operating state into modern state measurement [8].

Many PMUs are now being equipped with power stations and in industries around the developed countries. PMU is installed for numerous systems analysis such as, overload monitoring, protection and control of distribution generator, state estimation, power congestion management, and theft control [9]. The purpose of using PMUs in the power system is to prevent from catastrophic failures and provide real time measurement. Real time measurement can be obtained by using GPS antenna. PMUs role is important in the implementation of smart grid (SG) and it must be an essential part for SG in the future, therefore the amount of PMU installations is rapidly growing. The utmost important problems in the developing technology is the feasible site selection of metering device (PMU), the main reason is to limit the PMUs installation cost. Likewise, the communication cost that restricts the installation of PMUs with the other electrical parameters in the electricity grid, also the cost of communication network is somehow more than that of the PMUs device. Furthermore, site selection of PMU is also dependable on several different aspects, including the number of communication channels such as: Current Transformer (CT), Potential Transformer (PT) connection, connection of wiring to electrical components, station ground connection, and GPS Antenna connection [10]. Limitation of communication requirements and expensive nature of device have motivated researchers and power engineers to make a possible solution for the OPPP and find optimum location of PMU.

After assuring whole network observability, it is required to find ideal locations for the placement of PMU to maximize measurement redundancy. When the buses are observed by more than one PMUs it is called maximum redundancy of power system. In other words, the number of PMUs should be less than the number of buses and the arrangement of PMUs in a network should be in a proper order that devices should work as a backup for bus-bars.

There are several applications of PMU in power system, few earlier and recent PMU applications are discussed below. During the initial stages of PMU, it worked as a System Disturbance Recorders (DSDRs) due to the shortage of device and other practical limitations of bandwidth [11]. After US-Canada blackout in (2003), PMU device was used for post-mortem analysis of the incidents that happened during the outages of power supply. Optimal site selection for PMU placement intentions is to deeply observe all the buses in a grid station. Due to accurate monitoring of PMUs, it is useful in wide-area situational awareness. It monitors voltage behaviour and its trending. PMU measures the voltage phasors and current of all branches precisely which has a great impact on the state estimation performance [11].

This paper presents the optimization techniques which particularly work for the OPP problem. These proposed techniques are implemented for the desirable places of PMUs in power system or searching the minimal number of PMUs for entire network observability. Correspondingly, the paper gives a study about the recent work on OPP problem, what are the challenges and what are the achievements have been made through optimization algorithms. A typical Phasor Measurement Unit device is shown in Figure 1. For clarity, the paper has been organized into different section. The section 1 introduces the different optimization algorithms. While, section 2 describes the comparison results of optimization techniques, and section 3, 4 emphases on the conclusion and references for the optimum PMU placement. 


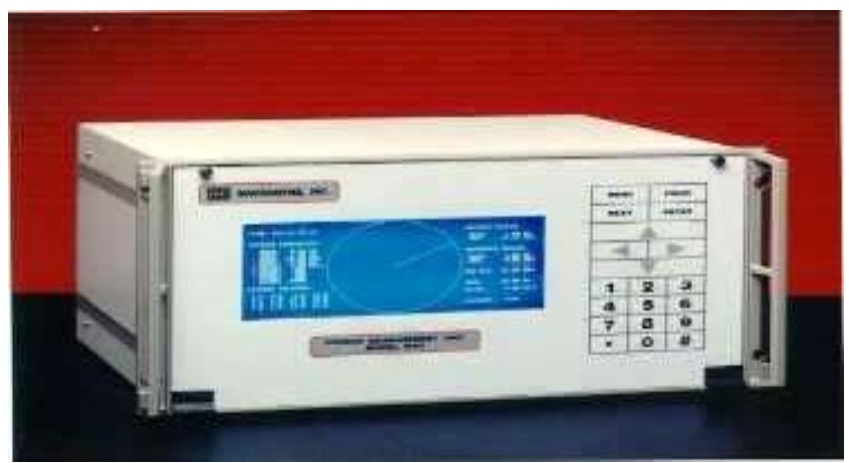

Figure 1. Phasor measurement unit (PMU) [12]

\section{OPTIMAL PLACEMENT METHODS}

The use of a computer program is more effective in choosing the best solutions from a given resources. There are few Mathematical algorithms such as, gauss probability and mathematical models which are playing their part in identifying the suitable results from available information. These methods are efficiently observing and assigning the best locations to rare resources which are known as optimization process. Furthermore, many mathematical optimization processes work for the PMU optimal locations namely, Greedy algorithm, Integer Linear Programming (ILP) and Integer Quadratic Programming. Similarly. Heuristic method is another member of optimization process which came into being when mathematical technique finds difficulty in searching the best solution. Moreover, this type of technique is frequently used to find weakness in the system and then improve the network with less amount of time. Few algorithms are categorised in Heuristic method, like Depth First Search (DEFS) and Minimum spanning tree (MST) which are explained in the next section.

Additionally, Metaheuristic is an optimization technique which performs better than both the above- mentioned techniques. It has higher processing and computationally speed which converges faster as compared to the other methods. Moreover, it is preferably used in finding the best alternatives from the available solution. Similarly, certain number of metaheuristic algorithms are grouped in simulated annealing (SA), genetic algorithm (GA), particle swarm optimization (PSO) are explained. Basically, optimization is involved in all the features around the world, which ranges from engineering technology to medical field, business marketing to construction designing. Furthermore, large amount of calculations can easily be solved in a micro seconds, which describes how important is the presence of these optimization techniques in finding the solutions.

\subsection{Mathematical programming methods}

Mathematical programming consists of matrix calculations and formulations through programs and equations. Most of the programs implemented on MATLAB of this category to solve OPP problem. Few important techniques are considered in the same approach namely, ILP, IQP and GA.

\subsubsection{Integer linear programming (ILP)}

A programme where all the defined parameters must use integer values is known as integer linear programming (ILP). An OPPP method is presented in [12], which aims to eliminate critical measurements from the entire network and make it into unnecessary information through optimal placement of PMU. This approach is helpful in detecting any bad data existence in real time measurement. Although, PMU measurement increases the capability of the system to detect and identify the critical data. Furthermore, this concept is implemented in the identification of suitable site selection of PMU which can lead to expose the fault or bad data in the measurement.

In [13], three different test cases have been used to simulate bad data, single PMU loss, and considers measurement of zero injection buses to minimize the amount of PMU with fully observable network. Furthermore, for the exact allocation of PMU devices, a systematically tactic is used in a low-cost perspective, in which binary (0-1) concept via ILP is addressed. Through simulation, it is shown that the importance of these devices in the power measurement is for bad data eradication as well as speedy computational analysis.

A general mathematical modelling for the OPP problem is presented in [14], which considers zero and without zero injection conventional measurement. The proposed method can save the system's computational time and can be used in a practical life. Simulation is carried out in different IEEE- standard test system for the allocation of PMU. IEEE standard test results show that small number of PMUs are placed in a smaller network for complete observability. 


\subsubsection{Integer quadratic programming (IQP)}

Quadratic programming $(\mathrm{QP})$ is an optimization procedure, in which only quadratic formulation is assured to solve the optimization problem. While during optimization it firstly converts the problem into quadratic model through mathematical programming. IQP method is used in [15], the main objective of this method is to reduce the required number of PMUs and provide maximum depth of measurement redundancy. To ensure the importance of IQP process, other optimization techniques are applied in the same literature for the OPP problem and then compared with the IQP. The idea of full network topological observability both for conditions, under normal operating conditions as well as single transmission line and single PMU outages are considered with the help of metrices criteria. Furthermore, formulation with conventional and without conventional measurement is used for different test systems by using N-1 possibility to find the alternatives for a single PMU and single line outages. The same formulation is involved in literature [16], the formulation is applied for the OPP problem to minimize the number of PMU and maximize observable buses. The uncertainty in the power system is reduced through synchronized PMU data. For the doubts in the measurement the RFV method has been used which is favourable for both random and non-random variation in the measurement.

\subsubsection{Greedy algorithm}

It is a simple in optimization process, which step by step selects the best optimal solution during the problem execution. Although, it is more limited in optimum results than the above two method which comes in the same category. In [17], two methods are proposed to reduce the size of the PMU placement and increase the computational performance. The first one is about removal of virtual information or the reduction of virtual buses. These buses increase the dimension of the system and the time of the optimization process, so it is necessary to eradicate the quantity of virtual buses from the system before starting the optimization. This approach can easily decrease the computational effort; besides it helps in finding the best optimal solution for OPPP with full network observability.

\subsection{Heuristic method}

Heuristic method uses graph searching technique to find the best solution. Two of the main algorithms of this methods are depth first search (DFS) and minimum spanning tree (MST). While, DFS is the old version of MST.

\subsubsection{Depth first search (DFS)}

DFS finds the optimal places for PMU using graph or tree search method, which search the best path without iteration. In [18], the PMU placement problem is solved using Power System Analysis Toolbox, which is an optimization-based software usually works in MATLAB/Simulink. DFS does optimization computationally by assigning places to PMU with a binary digit. For clarity, it assigns digit 1 to place PMU at a specific bus-bar and digit 0 shows that it does not take any interest in placing PMU in certain areas. In literature [19], results for the OPP problem are not optimum and ideal because DFS method operates speedily and finds the solution without iteration. It is experimentally proved with the comparison of other methods, one who does not search a result without iteration cannot provide better solutions. Likewise, DFS method uses greater number of PMUs in a smaller network such as, in 14-bus network it selects half of the area for the placement of PMU which is not economically good for power industries and networks.

\subsubsection{Minimum spanning tree (MST)}

Minimum spanning tree (MST) is an improved version of DFS method. MST enhances the performance of DFS in convergence and computational speed moreover, it improves searching ability. In [20] several simulations have been performed on IEEE-30-57 test system by MST on the china's Yunnan Power grid for optimal placement of PMU. MST indicates the exact location either it is faulty or suitable site selection of PMU. So, the characteristics of MST are added in SA technique for different projects to find the tempered area and involved in the applications of thermodynamics. Furthermore, the combination of SA with MST is used in the same literature, which improves the state estimation and wide area measurement performance for both SCADA and PMU measurements.

\subsection{Metaheuristic method}

Metaheuristic approach has several new techniques to handle with OPP problem. Such as, simulated annealing, genetic algorithm and particle swarm optimization are classified with this approach and these are much faster and more accurate than the older techniques. 


\subsubsection{Simulated annealing (SA)}

Simulated annealing (SA) technique is proposed for the combination and arrangements of an element, it has been implemented to keep continuity in the optimization. It is encouraged by the phenomenon of annealing cooling schedule in metallurgy, as it cools down the high temperature of materials which leads to a better global solution. During high temperature molecules have high energy to move randomly in a search space, thus an objective does not pay any attention in getting a best global result so, SA has an ability to ignore local results instead of global optima by using cooling schedule. The objective of this method is to process the data and identify the formation of lowest cost in the search space. It is also vital to control the updating process of PSO.

In literature [20], considers an installation cost of PMU using SA technique in order to provide stability, observability and consistency of the system for real time monitoring. The reduction in the installation cost of device is considered in order to maintain standard and quality of the state estimation.

The implementation/simulation results of the proposed method in IEEE-30 bus system shows how faster the average convergence speed is as compared to the other algorithms. To insure the quality of the algorithm several conformations are considered such as minimum cost, number of critical measurement and number of critical measurement and sets. Which is proved by capturing and then eliminating lots of critical measurement and size of the device, which is achieved by very fast computational period.

The objective of SA techniques in [21], is to provide the minimum places for PMU and exposes the dynamic information in the system at the same time. The variation in the information is obtained by the analysis of the topological observability which does not only help in measuring the complex constraints but also provide preliminary placement for PMU. Furthermore, Incidence matrices rule based on topological observability is applied for spanning graph to allot the best areas for PMU with more incident branches in unobservable region. Correspondingly, in this literature different sensitivity analysis method using SA has been applied on IEEE-14 bus network, which cannot only search the desire locations for PMU but also measure the intuitive parameters at the identical period.

A convincing SA technique is adopted in [22], where the issue with the site selection of PMU is solved by using the capacity of basic constraints. Another objective of this literature is based on the measurement of SCADA and PMU, which is used to control all the state variables for power flow estimation without repetition. Without iteration means load flow estimation (voltage and phase angle) can be observed without calculation. Furthermore, a reduction scheme concept is applied to reduce the graph into tree, which breaks the scheme loop into tree such as, those buses which have maximum number of incident branches are removed in order to break the scheme loop into tree. Once the incident branches are removed from the loop arrangement, which results in making the bus terminals that helps to continue the construction of tree. Practical results on IEEE-13 bus network describe that SA method calculates the state parameters or state problem computationally very fast. Thus, no additional PMU is required for the same network.

An improved SA (ISA), algorithm is presented in [23], the main objective of this method is to make convergence faster and diminish the searching process with high computational speed by the comparison of simple SA algorithm, likewise:

a) Amendment in the existing parameters (current temperature and cooling schedule) in which current state can be considered to increase the convergence reliability.

b) As to decrease the length of the searching area, direct combination (DC) approach is considered using an actual heuristic procedure to pick the main sets for the observability.

c) The same heuristic rule is used in Tabu search (TS) in order to find the ideal result and to maintain the searching area.

\subsubsection{Genetic algorithm (GA)}

A genetic algorithm (GA) is an adaptive heuristic process based on the evolutionary concepts of the natural selection. It is an optimization technique which searches the problem and selects best possible outcome from the available resources. In [24], it recommends a use of GA method with different conditions of PMU placement to find the solution for OPPP. The PMU measurements are used in state estimation to identify the bad data and provide reliable observability of the system. The authentication of the proposed method has been proved through simulation on IEEE-14 test system and Russian Regional electrical power network. The tabulated results are obtained from the simulation, which shows the improvement in the efficiency of state estimation through the measurement of PMU.

An improvement in the accuracy of estimator with the measurement of conventional and PMU using GA method is described in [25], This literature considers placement of PMU device as an optimization problem. When SE uses PMU data its equation becomes linear, for a larger power network PMU data is not enough for SE equation to make it linear. Due to this reason with the combination of traditional approach and PMU measurement using GA method is considered to solve this issue. Objective of the proposed method is to manage 
the congested region and predict it, based on the synchronized measurement by PMU. The simulation results are presented with the 3D- illustration for better resolution.

To ensure the topological observability of the power network a genetic algorithm is presented in [26], in which GA controls the placement and distribution of PMU device with the obtained phasor measurement by PMUs. The characteristic of the presented work is to present designated programming code for each chromosome in the population. The programming is carried out by encoding, in which chromosome represents the connectivity of the power network. These coding clearly figure out and estimate the fitness function for each objective. Also, the fitness value of each individual and the reproduction of chromosome crossover, mutation, and different steps are performed. Furthermore, to solve the placement problem of PMU, the simulation has been verified on 4-IEEE standard test system. The flow chart of GA is depicted in Figure 2.

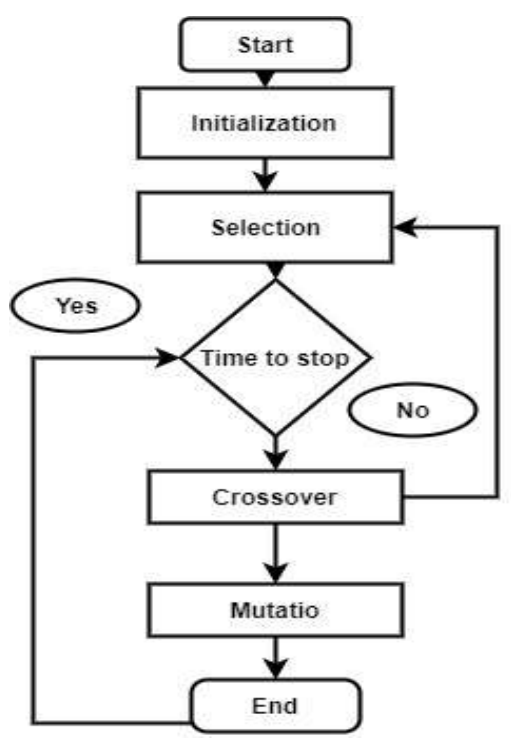

Figure 2. Flowchart for genetic algorithm [27]

\subsubsection{Particle swarm optimization (PSO)}

Particle swarm optimization (PSO) was proposed by Russell Eberhart and James Kennedy in 1995, which is also associated with metaheuristic techniques. The operation of this method is totally matched with the searching ability of birds and fishes, as they find food in a group shape for better communication. It is a population-based algorithm, in which every individual called particles such as a collection of particles in a search space is known as population. Furthermore, the concept of PSO defines, every individual/particle travel towards best fitness value in a search spaces with updating velocity and position. During changing position these particles not only store their own position and velocity but also store their neighbour's data and information at every iteration. The variation in the position and velocity of these particles' nature, describe the best fitness value which is upgraded and stored after every iteration.

An improved binary version of PSO is proposed in [27]. To reduce the number of PMUs and provide full network observability a BPSO is considered as an optimization tool. Different rules are applied based on network graph procedure through topological analysis calculation. Different groups of unknown buses are observed with the consideration of node equation and ZIB. Moreover, for obtaining the maximum depth of voltage phasor measurements few buses are eliminated from the network. Only those buses are eliminated from the network such as, zero injection buses (no load, no generation), and especially those buses which do not consist more than one incident current branches. This approach has helped in reducing the number of PMUs installation and a searching space.

In reference [28], the novelty of the presented work is to reduce the PMU installation cost. Which describes about the different areas have dissimilar impact on installation cost. Likewise, the installation cost of PMU varies according to the selection of area. In this work, a test is conducted on 17-bus system by setting the conditions (population size, iteration counter etc.). Result of this presented method is compared with the conventional method, which clearly describes that the allocation of PMUs in different areas by proposed technique improves cost factor than the traditional approaches. However, cost factor is varied in conventional method with different locations of PMU. 


\section{PERFORMANCE COMPARISON OF DIFFERENT ALGORITHMS}

Different optimization algorithms are compared with their performances in Table 1 . The characteristics of these techniques are varied according to their nature. Almost every algorithm has different characteristics from one another when they perform their operation. Whereas, the data obtained from the provided literatures shows that which method is suitable for the optimal placement of PMU and optimization processes. Nevertheless, the Table 1 also describes that which algorithm has low performance ratio. Likewise, the system observability and computational efficiency of GA is averaged but the requirement of PMU is low. Similarly, the performances of the other algorithms are below than the average which require more processing time to execute the program. Linear integer programming does not provide proper systems' observability in terms of PMU placement. It is clearly observed from the literatures, that the attributes of the PSO algorithm regarding its performance is much better than the existing optimization techniques.

Table 1. Comparison between different OPP method

\begin{tabular}{|c|c|c|c|c|c|c|}
\hline Ref & Algorithms & $\begin{array}{l}\text { Computational } \\
\text { Efficiency }\end{array}$ & $\begin{array}{c}\text { System } \\
\text { Observability }\end{array}$ & $\begin{array}{c}\text { Computational } \\
\text { Time } \\
\end{array}$ & $\begin{array}{c}\text { System } \\
\text { Complexity }\end{array}$ & $\begin{array}{c}\text { PMU } \\
\text { Requirements }\end{array}$ \\
\hline$[15]$ & Integer linear programming (ILP) & Moderate & Low & Low & High & High \\
\hline [23] & Simulated annealing (SA) & High & High & Low & Low & High \\
\hline [25] & Genetic algorithm (GA) & Moderate & High & Moderate & Moderate & Low \\
\hline [28] & Particle swarm optimization (PSO) & High & Moderate & Moderate & High & Moderate \\
\hline
\end{tabular}

\section{CONCLUSION}

For solving the problem of OPP many optimization techniques have been presented and reviewed. The aim of these techniques is to minimize the number of installed PMUs and maximize the observable buses under normal operating conditions. The validity and sustainability of these techniques are verified through simulations and implementation in different practical power grids and transmission network. However, these optimization methods have both pros and cons during operation. Such as, few of them have low computational efficiency and few of them have high performance rate. Consequently, from the research study it is found that the PSO algorithm works better than the existing optimization techniques and it will be more useful in the future work. This paper will provide a platform to power engineers and researchers to select best technique while working on OPP problem in the future work. Also, it will help in sorting different techniques for OPP problem and finding an appropriate result. The presented optimization methods are valid for solving the complex issues which make easiness in upcoming efforts for optimal placement of PMU and will bring stability and reliability in the power system.

\section{REFERENCES}

[1] C. Mishra, "Modelling of Phasor Measurement Unit and Phasor Data Realisation with 2 Bus System," Intl J Engg Sci Adv Res., vol. 1, no. 2, pp. 3-8, 2015.

[2] M. S. Shahriar, I. O. Habiballah, and H. Hussein, "Optimization of phasor measurement unit (PMU) placement in supervisory control and data acquisition (SCADA)-based power system for better state-estimation performance," Energies, vol. 11, no. 3, 2018.

[3] Jitender Kumar, "Impact of Phasor Measurement Unit on The State Estimation of Large Power System," international journal of advanced research and innovation, UP, india, pp. 1-7, 2017.

[4] A. Waqar, Z. Khurshid, J. Ahmad, M. Aamir, M. Yaqoob, and I. Alam, "Modeling and simulation of phasor measurement unit (PMU) for early fault detection in interconnected two-area network," Proc. - 2018, IEEE 1st Int. Conf. Power, Energy Smart Grid, ICPESG 2018, pp. 1-6, 2018.

[5] L. Sun et al., "Optimum Placement of Phasor Measurement Units in Power Systems," IEEE Trans. Instrum. Meas., vol. 68, no. 2, pp. 421-429, 2019.

[6] P. W. Davis, “Apparatus Systems,” no. 4, pp. 1534-1542, 1980.

[7] and D. L. Yang Gao, Zhijian Hu, Xixiong He, "Optimal placement of PMUs in power systems based on improved PSO Algorithm,” 2018 3rd IEEE Conf. Ind. Electron. Appl., no. 2, pp. 2464-2469, 2018.

[8] S. P. Pokharel and S. Brahma, "Optimal PMU placement for fault location in a power system," 41st North Am. Power Symp. NAPS 2009, pp. 1-5, 2009.

[9] R. F. Nuqui and A. G. Phadke, "Phasor measurement unit placement techniques for complete and incomplete observability," IEEE Trans. Power Deliv., vol. 20, no. 4, pp. 2381-2388, 2005.

[10] \& N. Paolone, M., Borghetti, A., "Paolone, Mario, Alberto Borghetti, and Carlo Alberto Nucci. "A synchrophasor estimation algorithm for the monitoring of active distribution networks in steady state and transient conditions.," Proc. 17th Power Syst. Comput. Conf. (PSCC 2011, vol. 17, no. 2011, pp. 1-8, 2011.

[11] D. F. Hoeschele, “Analog-to-digital and digital-to-analog conversion techniques," IEEE J. Solid-State Circuits 25, vol. 968, no. 1994 Apr, pp. 127-128, 1994. 
[12] J. Chen, S. Member, and A. Abur, "Placement of PMUs to Enable Bad Data Detection in State Estimation," IEEE Transactions on Power Systems, vol. 21, no. 4, pp. 1608-1615, 2006.

[13] X. Bei, Y. J. Yoon, and A. Abur, "optimal placement and utilization of phasor measurements for state estimation," PSCS, no. August, pp. 22-26, 2005.

[14] B. Gou, "Generalized Integer Linear Programming Formulation for Optimal PMU Placement," IEEE Transactions on Power Systems, vol. 23, no. 3, pp. 1099-1104, 2008.

[15] S. Chakrabarti and E. Kyriakides, "Placement of Synchronized Measurements for Power System Observability," IEEE Transactions on Power Delivery, vol. 24, no. 1, pp. 12-19, 2009.

[16] S. Chakrabarti, E. Kyriakides, M. Albu, and S. Member, "Uncertainty in Power System State Variables Obtained Through Synchronized Measurements," IEEE Trans. Instrum. Meas., vol. 58, no. 8, pp. 2452-2458, 2009.

[17] M. Zhou et al., "A Preprocessing Method for Effective PMU Placement Studies," 2008 Third International Conference on Electric Utility Deregulation and Restructuring and Power Technologies, no. April, pp. 2862-2867, 2008.

[18] M. Farsadi, H. Golahmadi, and H. Shojaei, "Phasor Measurement Unit (PMU) allocation in power system with different algorithms," 2009 Int. Conf. Electr. Electron. Eng., no. c, pp. 396-400, 2009.

[19] T. Cai and Q. Ai, "Research of PMU Optimal Placement in Power Systems," vol. 2005, pp. 38-43, 2005.

[20] Y. Yang, H. Shu, and L. Yue, "Engineering Practical Method for PMU Placement of 2010 Yunnan power grid in China," 2009 Int. Conf. Sustain. Power Gener. Supply, pp. 1-6, 2010.

[21] Z. Hong-shan, L. Ying, M. Zeng-qiang, and Y. Lei, "Sensitivity Constrained PMU Placement for Complete Observability of Power Systems," 2005 IEEE/PES Transmission \& Distribution Conference \& Exposition: Asia and Pacific, pp. 1-5, 2005.

[22] A. M. Glazunova, I. N. Kolosok, and E. S. Korkina, "PMU placement on the basis of SCADA measurements for fast load flow calculation in electric power systems," 2009 IEEE Bucharest PowerTech, pp. 1-6, 2009.

[23] S. H. Hyun, "Optimal placement of phasor measurement units with GPS receiver," vol. 00, no. 2, pp. 258-262, 2001.

[24] A. Z. Gamm, I. N. Kolosok, A. M. Glazunova, and E. S. Korkina, "PMU placement criteria for EPS state estimation," 3rd Int. Conf. Deregul. Restruct. Power Technol. DRPT 2008, no. April, pp. 645-649, 2008.

[25] M. Gavrilaş, I. Rusu, G. Gavrilaş, and O. Ivanov, "Synchronized phasor measurements for state estimation," Électrotechnique Et Électroénergétique, pp. 335-344, 2008.

[26] F. J. Marín, F. García-Lagos, G. Joya, and F. Sandoval, "Optimal Phasor Measurement Unit Placement using Genetic Algorithms," International Work-Conference on Artificial Neural Networks, pp. 486-493, 2007.

[27] M. Hajian, A. M. Ranjbar, T. Amraee, and A. R. Shirani, "Optimal placement of phasor measurement units: Particle swarm optimization approach,” 2007 Int. Conf. Intell. Syst. Appl. to Power Syst. ISAP, no. 1, 2007.

[28] C. Su and Z. Chen, "Optimal placement of phasor measurement units with new considerations," Asia-Pacific Power Energy Eng. Conf. APPEEC, pp. 1-4, 2010. 